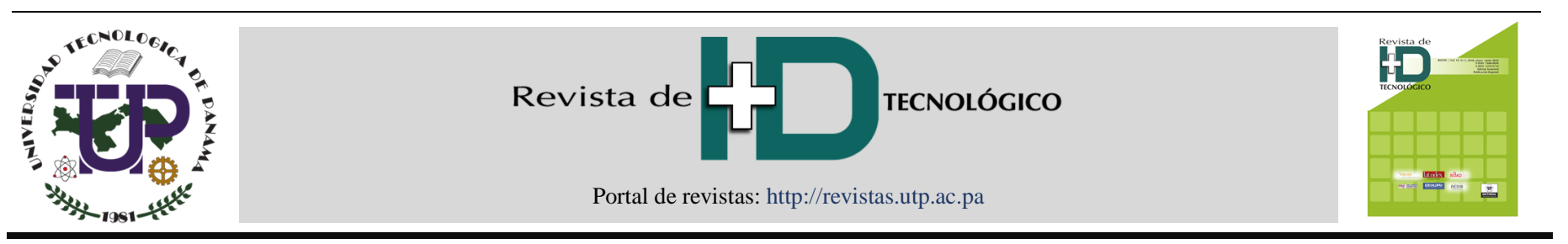

\title{
Caracterización de imaginación motora utilizando análisis de descomposición de bandas de energía
}

\section{Characterization of motor imagination using decomposition analysis of energy bands}

\author{
I. Moreno ${ }^{1 *}$, C. Boya ${ }^{2}$, J. Serracín ${ }^{1}$, S. Serracín ${ }^{1}$, E. Batista ${ }^{1}$, J. Antelis ${ }^{3}$, L. Hernández ${ }^{3}$, R. Moreno ${ }^{1}$, L. Gómez ${ }^{1}$, J. Quintero ${ }^{1}$ \\ ${ }^{1}$ Grupo ROBOPROC, Centro Regional de Chiriquí, Universidad Tecnológica de Panamá, Panamá \\ ${ }^{2}$ Universidad Interamericana de Panamá, Panamá \\ ${ }^{3}$ Extensión de Guadalajara, Instituto Tecnológico de Monterrey, México \\ *Autor de correspondencia: iveth.moreno@utp.ac.pa
}

\begin{abstract}
RESUMEN- Este artículo trata sobre el análisis de las señales eléctricas generadas por el cerebro, cuando una persona imagina el movimiento de sus extremidades superiores. Las señales que se estudian son adquiridas con un equipo de interfaz cerebro-computador y son analizadas utilizando la transformada Wavelet Discreta para la técnica de descomposición de banda de energía. El uso de esta técnica es novedoso en el análisis de las señales eléctricas generadas por el cerebro y los resultados que se obtuvieron indican que el uso de la técnica es factible para la caracterización de señales eléctricas provenientes del cerebro.
\end{abstract}

Palabras clave-Bandas de energía, cerebro, EEG, imaginación motora, interfaz cerebro-computadora, wavelet.

\begin{abstract}
This article provides an overview of the analysis of electrical signals generated by the brain, when a person imagines the movement of their upper extremities. The signals studied are acquired with a brain-computer interface equipment and analyzed using Discrete Wavelet transform as the energy band decomposition technique. The use of this technique is new in the analysis of electrical signals generated by the brain and the results obtained indicate that the use of the technique is feasible for the characterization of electrical signals coming from the brain.
\end{abstract}

Keywords-Energy band, brain, EEG, motor imagination, brain-computer interface, wavelet.

\section{Introducción}

En este documento se aborda el uso del análisis de bandas de energía mediante transformada discreta de Wavelet, como posible técnica para la caracterización de señales de electroencefalografía (EEG) provenientes del cerebro como resultado de la imaginación motora. Las señales son obtenidas por medio de una interfaz cerebro-computadora, también conocida como BCI (brain computer-interface). Para realizar este estudio, se utilizaron datos obtenidos con un equipo g.Nautilus BCI de ocho canales.

Se define una interfaz cerebro-computadora, como un sistema que mediante los avances en técnicas de neurociencia, procesamiento de señales, machine learning y tecnología de la información [1], logra medir la actividad del sistema nervioso central y traduce la lectura de estas señales (EEG, ECoG, etc.) en "salidas artificiales" (brazos robóticos o sillas de ruedas), que les permiten a las personas reemplazar, restaurar, aumentar, suplantar o mejorar las "salidas naturales" que controla el sistema nervioso central del cuerpo humano (extremidades superiores e inferiores) [2].

El artículo seguirá la siguiente estructura: primera sección introducción, segunda sección antecedentes, tercera sección materiales y métodos, cuarta sección análisis de resultados, quinta sección conclusiones y discusión y por último en la sexta sección las referencias bibliográficas.

\section{Antecedentes}

La tecnología BCI no siempre fue una realidad tangible, incluso para la mayoría de científicos en los años sesenta, no era más que "ciencia ficción" que se veía en películas o la televisión. Como muchas otras tecnologías que antes el ser humano solo podía soñar en crear y utilizar, los sistemas de interfaz cerebro-

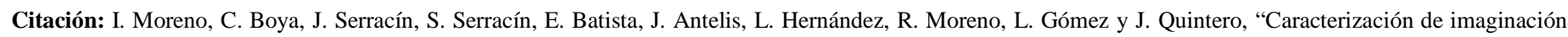
motora utilizando análisis de descomposición de bandas de energía”, Revista de I+D Tecnológico, vol. 15, no. 1, pp. (71-79), 2019.

Tipo de artículo: Original Recibido: 24 de agosto de 2018. Recibido con correcciones: 3 de septiembre de 2018 . Aceptado: 29 de enero de 2019.

DOI https://doi.org/10.33412/idt.v15.1.2101

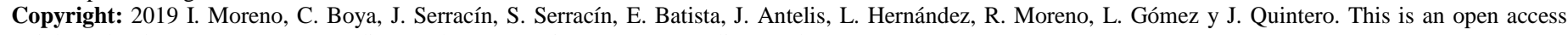
article under the CC BY-NC-SA 4.0 license (https://creativecommons.org/licenses/by-nc-sa/4.0/). 
computadora hoy en día son una realidad gracias a los avances en las diversas disciplinas afines con este campo de investigación.

Fue en los años veinte cuando el precursor Hans Berger [3] realizó los primeros EEG. De este modo, científicos de todo el mundo comenzaron a preguntarse por los diferentes posibles usos para esta nueva tecnología, como por ejemplo comunicación u otras acciones sin la necesidad de mover músculos, solo por medio de ondas cerebrales [2].

No es hasta 1964 cuando el médico Grey Walte, describe y logra hacer funcionar con aparente éxito el primer sistema BCI, pero sin publicaciones oficiales al respecto [4]. Actualmente hay un sin número de personas desarrollando esta área de estudio, manteniendo como principal aplicación o propósito mejorar la calidad de vida de personas con discapacidad motora [5] [6] [7], al igual que debido a los avances de la tecnología y la reducción de costos para esta clase de equipos se ha dado lugar a otras aplicaciones como por ejemplo: prevenir y reducir el deterioro cognitivo en personas de edad avanzada [8], rehabilitación de personas con discapacidad de movimiento por medio de simulaciones [9], videojuegos [10], control de sillas de ruedas en entornos virtuales [2], entre otros.

Las diferentes etapas elementales de un sistema BCI son: adquisición y procesamiento de señales, generación de comandos y control de salidas; y finalmente retroalimentación al usuario en tiempo real. Como se puede observar en la figura 1.

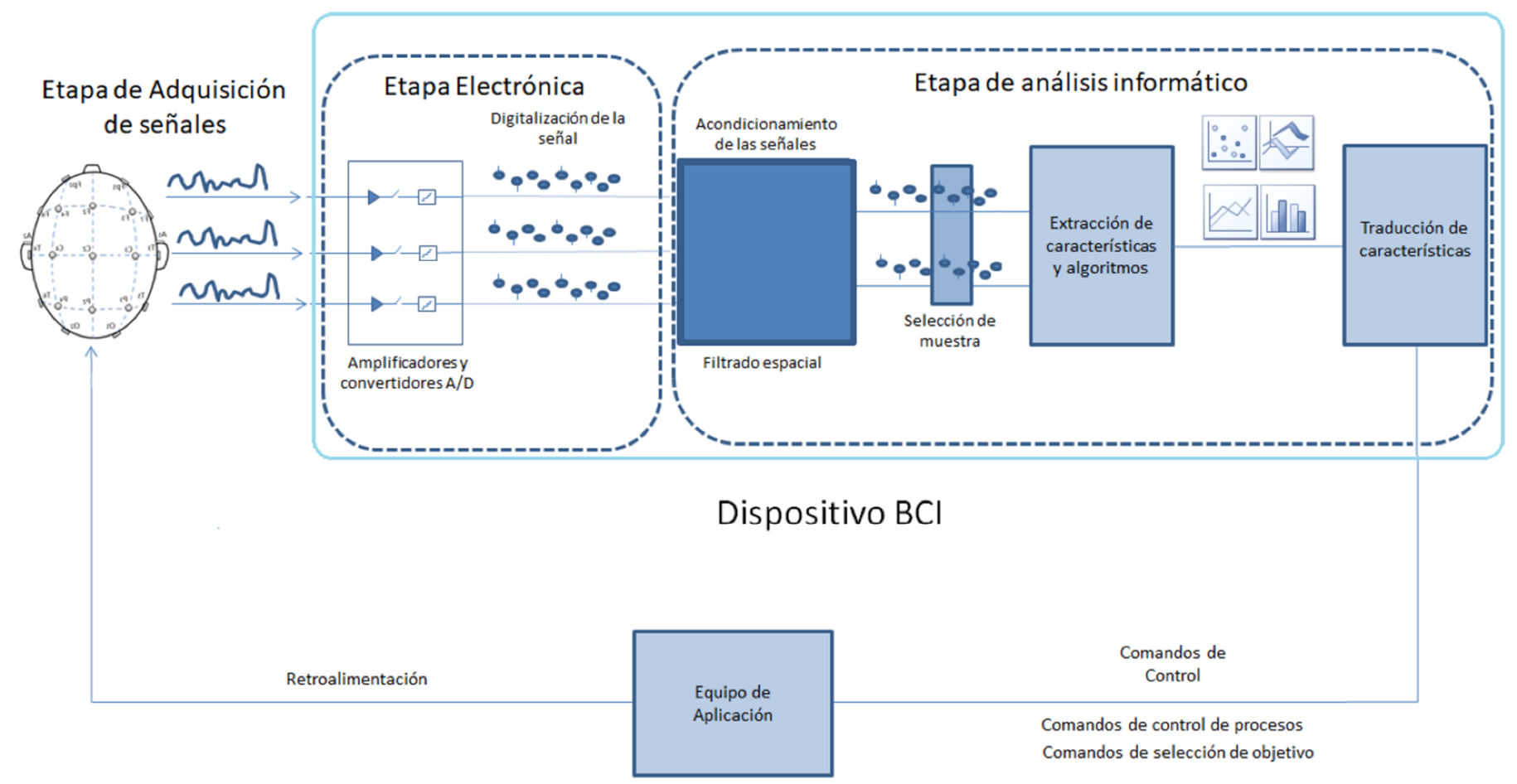

Figura 1. Diagrama de etapas en un sistema BCI.

\subsection{Adquisición y procesamiento de señales}

En esta etapa se obtienen las señales eléctricas generadas por el cerebro, ya sea de forma invasiva por medio de cirugía o no invasiva por medio de electroencefalografía (EEG) [4]. Este último es el método más utilizado y económico del mercado.

La colocación de estos sensores a lo largo del cuero cabelludo es según el Sistema Internacional 10-20, se pueden utilizar desde 8 hasta 64 sensores [2].
Por otro lado, el procesamiento de las señales incluye hasta tres subetapas: preprocesamiento, extracción y detección y clasificación de características [4].

El preprocesamiento consiste en mejorar la calidad de la señal para facilitar su posterior análisis, aquí por ejemplo, se encuentran diferentes sistemas de filtrado; ya sea en el mismo sensor o en equipos exclusivos de filtrado de señal. Las señales se pueden contaminar con 
diferentes agentes externos, como el campo magnético generado por la electricidad de alimentación del equipo $(120 \mathrm{~V} 60 \mathrm{~Hz})$, entre otros. Con esto se busca obtener un mejor SNR (signal-to-noise ratio), para así alterar en lo mínimo posible los datos inherentes en la señal original [2].

En la extracción de características se utilizan algoritmos especiales para "calcular o extraer" estos parámetros, basándose en diferentes frecuencias según la actividad mental que se esté ejecutando, cantidad de actividad en determinados sectores del cerebro, niveles de energía en sensores específicos, etc.

Y por último en la detección y clasificación de características, por medio de programas especializados se agrupan los diversos parámetros de interés según características particulares, cabe destacar que si se lleva un control de tiempo sobre las tareas mentales a realizar por el usuario se facilita esta subetapa del proceso; luego se traducen o transforman estas características en comandos específicos de control.

\subsection{Generación de comandos y control de salidas}

Existen dos tipos de procesos para generar comandos de control en BCI, comandos que seleccionan un objetivo y comandos que controlan un proceso [2]. Cuando se trabaja con sistemas BCI sencillos, donde las variables de salida y entrada son pocas, se recomienda utilizar comandos de selección debido a su poca complejidad en comparación de los comandos de control de procesos.

Cuando se habla de comandos de selección de objetivos, se entiende que el usuario debe pensar en la acción final que desea realizar (por ejemplo, levantar una prótesis de brazo); y es un programa dentro del brazo el que toma las decisiones para realizar el movimiento. Por el contrario, cuando se habla de comandos de control de procesos, el usuario es el que debe tomar todas las decisiones para conseguir el objetivo final (por ejemplo, el usuario tendría que decir que tan rápido va a mover el brazo, que tanto desea doblar el codo, etc.); y el brazo robótico solo llevaría a cabo las acciones de mover los motores en las direcciones señaladas por la persona.

\subsection{Retroalimentación}

Es muy importante que se realice una retroalimentación en tiempo real de los resultados obtenidos con los comandos de control sobre los actuadores según sea la aplicación BCI. Es necesaria, ya que de este modo el usuario y quien realiza las pruebas, pueden comprobar si se pudo llegar con éxito a su objetivo o si hay que realizar cambios en algún punto del proceso.

En resumen, BCI se basa en la posibilidad de medir la actividad eléctrica que existe en el cerebro, su procesamiento y salida para la interacción del cerebro con el exterior.

Por otro lado, el concepto de electroencefalografía (EEG) hace referencia a una técnica de adquisición de la señal eléctrica cerebral de forma no invasiva; en la que se registra la región emisora, la polaridad y los cambios espacio-temporales de dichas señales, por medio de electrodos superficiales y basales que transforman las corrientes iónicas del tejido cerebral en corrientes eléctricas en la superficie craneal.

Hay varios sistemas para el posicionamiento de los electrodos, como lo son: Illinois, Montreal, Aird, Lennox, Marchall, etc. [11]. Pero en 1958 fue propuesto por la Federación Internacional de Sociedades de Electroencefalografía y Neurofisiología Clínica el uso estandarizado del Sistema Internacional diez-veinte, mencionado anteriormente (ver figuras 2 y 3 ).

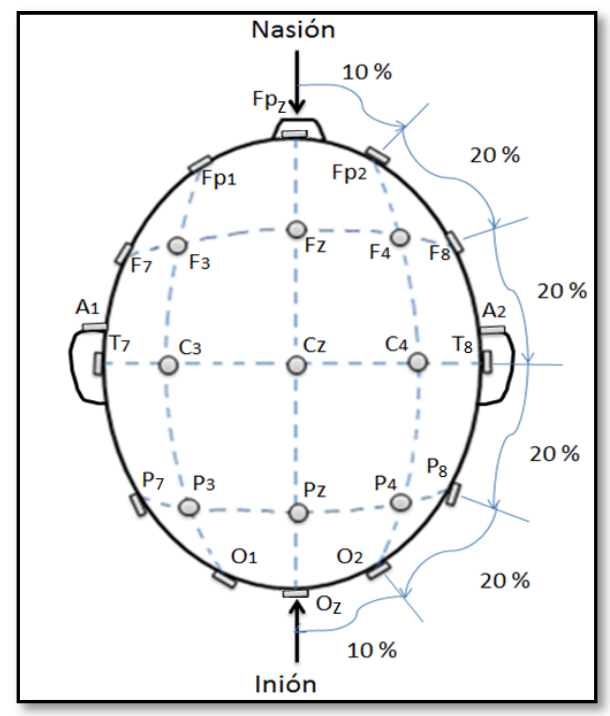

Figura 2. Diagrama del Sistema Internacional 10-20, vista superior. 


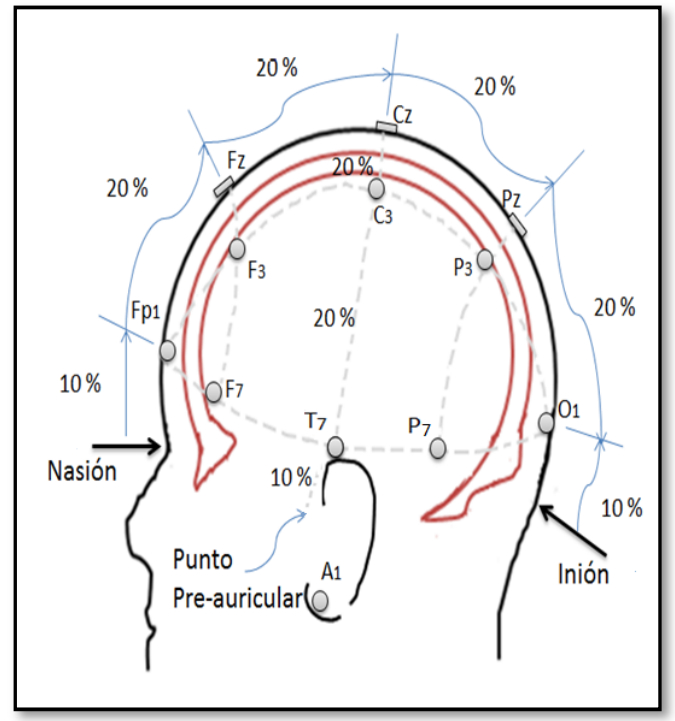

Figura 3. Diagrama del Sistema Internacional 10-20, vista lateral izquierda.

Al mismo tiempo, las señales obtenidas se pueden estudiar, utilizando diferentes técnicas como son: filtros, en conjunto con transformadas como Fourier y/o redes neuronales. La finalidad es que mediante la aplicación de una u otra técnica matemática puedan representarse parámetros únicos en la señal, que puedan dar indicio o diferenciar actividades cerebrales de distinta naturaleza.

El cerebro por su naturaleza genera señales con frecuencias variables a lo largo del tiempo, por lo que la aplicación de un método matemático como la transformada de Wavelet, la cual opera con frecuencias variables de eventos en el tiempo [12] puede ser efectivo.

La distribución de energía promedio, es un método grafico - matemático, mediante el cual se observa el comportamiento energético de las señales obtenidas mediante electroencefalograma (EEG), de acuerdo a las frecuencias que presenta dichas señales. Este método en general, no es utilizado de forma directa, sino, como una etapa de preprocesado, para ser enviado por ejemplo a una red neuronal. Ejemplo de esto, en el estudio realizado en [13], por medio de reconocimiento de voz humana se pretende clasificar entre género masculino o femenino. Por otro lado, en [14], el propósito es la identificación de señales anormales en el cerebro, para determinar signos de epilepsia utilizando electroencefalograma (EEG). Además, en [15], se muestra la distribución de energía con la frecuencia de cada tipo de descarga y la desviación estándar, utilizando distribución promedio de energía. En el caso de este estudio, se propone utilizar el método directamente para discriminar de un pensamiento sobre otro, obtenido por medio del electroencefalograma (EEG).

El cerebro por su naturaleza genera señales con un espectro que va desde menos de 1 Hertz hasta los 100 Hertz. Dependiendo de la actividad detectada, ya sea en tiempo y espacio, algunas frecuencias presentan más energías que otras. Cuando esta actividad está presente debido a una intensión muy enfocada por parte del usuario, las señales EEG generadas tienen una huella distintiva de energía en frecuencia. Aunque actualmente se utiliza la técnica de transformada de Fourier para realizar análisis y extracción de características en frecuencia, esta técnica genera un espacio de características del tamaño de la señal en el tiempo. En este documento, se utiliza una técnica basada en la transformada Wavelet para extraer características de la señal proveniente de la imaginación motora [13][14][15]. La transformada Wavelet, muestra la relación que hay entre la onda objeto de análisis y un conjunto de ondas especiales con características peculiares de amplitud y frecuencias; el resultado final sería la suma de todos los valores obtenidos al variar las distintas características de dichas ondas. Se observa el cómo varía o responde una función madre al estar en convolución con las ondas wavelet; y en base a la resultante obtener coeficientes [16]. Esta transformada es utilizada para obtener una "huella" de energía más compacta y representativa de las señales EEG generadas por la intensión del usuario [12]. El objetivo es generar huellas de energía que corresponden a cuatro acciones básicas, imaginación de levantamiento de brazo izquierdo, imaginación de levantamiento de brazo derecho, parpadeo y descanso. Las huellas se crean a partir de múltiples muestras efectuadas de las acciones que se mencionan y se comparan entre sí a fin de establecer conglomerados de niveles de energía. Los conglomerados corresponden a la huella de energía y se obtienen valores muy característicos para cada acción.

\section{Materiales y métodos}

La fase experimental de este estudio consistió en adquirir datos de electroencefalografía, producto de estimulación visual a personas sanas a que realizaran 
tareas de imaginación motora del movimiento de las extremidades superiores. Cada persona imaginó una rutina de pensamientos durante dos minutos y 24 segundos, de la siguiente forma: descanso, movimiento de la mano derecha o izquierda de forma aleatoria; y "parpadeo". Es importante aclarar que el pensamiento parpadeo va asociado, también a una acción física de mover las pestañas repetidamente. Esta acción permite tener un marco de referencia, entre pensamientos y una acción, que puede ser observada más claramente en las gráficas y para comodidad del usuario, ya que durante los otros pensamientos no puede pestañear para no contaminar la señal. Cada pensamiento de descanso o movimiento de mano derecha/izquierda o de parpadeo tiene una duración de cinco segundos y el conjunto: descanso, movimiento derecha o izquierda y parpadeo; 15 segundos, en el video se repetía dicha secuencia 9 veces. El objetivo de estas tareas es la obtención de señales EEG desde varios pensamientos, buscando extraer las características de las señales con la técnica de descomposición Wavelet.

Para adquirir los datos, se procedió a colocar el gorro BCI sobre la persona sana (ver la figura 4). El gorro utilizado posee una cinta para ajuste de tamaño en la barbilla; mediante este ajuste de la cinta, se fija la posición del equipo, de forma que no se distorsionen los datos por deslizamientos, que suele ser una de las principales causas físicas de alteración de los datos. Una vez con el sistema correctamente fijado la persona entonces mira en una pantalla las palabras Descanso, Movimiento Derecha o Izquierda, Parpadeo, a medida que aparecen, indicando a la persona con dichas palabras la instrucción en que debe pensar, dentro de un ambiente controlado.

El gorro BCI contiene ocho electrodos colocados de acuerdo al Sistema 10/20. Las señales eléctricas obtenidas a través de los electrodos son enviadas a una etapa amplificadora y de filtrado, para luego enviar la señal a la computadora, donde se analizarán las señales obtenidas.

El objetivo de este estudio es evaluar la efectividad del método para la identificación de eventos, con intención de diferenciar entre la imaginación de movimiento del brazo izquierdo o la imaginación de movimiento del brazo derecho.

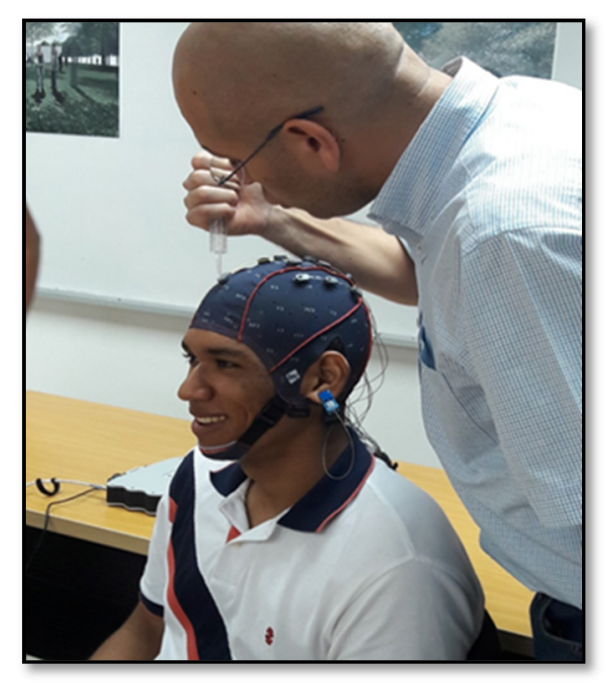

Figura 4. Sistema BCI no invasivo de 8 canales.

\section{Resultados}

En la figura 5 se muestran las señales en el estado bruto de los datos, para dos de los participantes; donde la figura superior es un participante y la figura inferior es otro participante. Se observa que la forma de ambas señales mantiene casi la misma apariencia para ambos participantes, con diferencias en el canal 1 y el canal 8 . En este caso, el sistema requiere de más pruebas para determinar el porqué de esta diferencia.

Por otro lado, en la figura 6, se observa el estado de calma en un participante, ya sea con los ojos abiertos o cerrados. Para ambos casos, la señal presente en cada sensor, mantiene un tipo de onda similar.

En la figura 7 se muestran las señales EEG del participante A, para los 8 canales, y en donde los números $1,2,3$ y 4 superiores son las intenciones de imaginación motora que se le ha solicitado al participante A, donde 1 es el estado "Calma", 2 el estado "mover a la derecha", 3 el estado "mover a la izquierda" y el 4, "parpadeo". Este último se utiliza para tener una referencia más clara entre cada prueba. Cada prueba se inicia con el estado de "Calma" y después con el estado de movimiento, ya sea para la izquierda o la derecha. Cada uno de estos estados tiene una duración de cinco segundos, siendo cada prueba de 15 segundos en total. Se realizan nueve pruebas por cada sesión. En la figura 5, por claridad, se ha dividido con líneas de referencia azules cada estado de cinco segundos. De los cuatro estados, se observa que el estado "parpadeo" es una variación relativamente rápida, lo cual es 
proporcional al movimiento físico de cerrar los ojos rápida y repetidamente. Por otro lado, observando cada estado, es difícil visualmente diferenciar entre los estados calma y movimiento; y más aún, entre los movimientos derecha e izquierda. Con el fin de lograr esta discriminación, se aplica la técnica de descomposición Wavelet, la cual permite obtener niveles de energía por banda de frecuencia. Como se conoce la señal que corresponde a cada uno de los estados, estos son procesados por la descomposición Wavelet, obteniendo la energía de una serie de bandas de frecuencias como se muestra en la tabla 1.
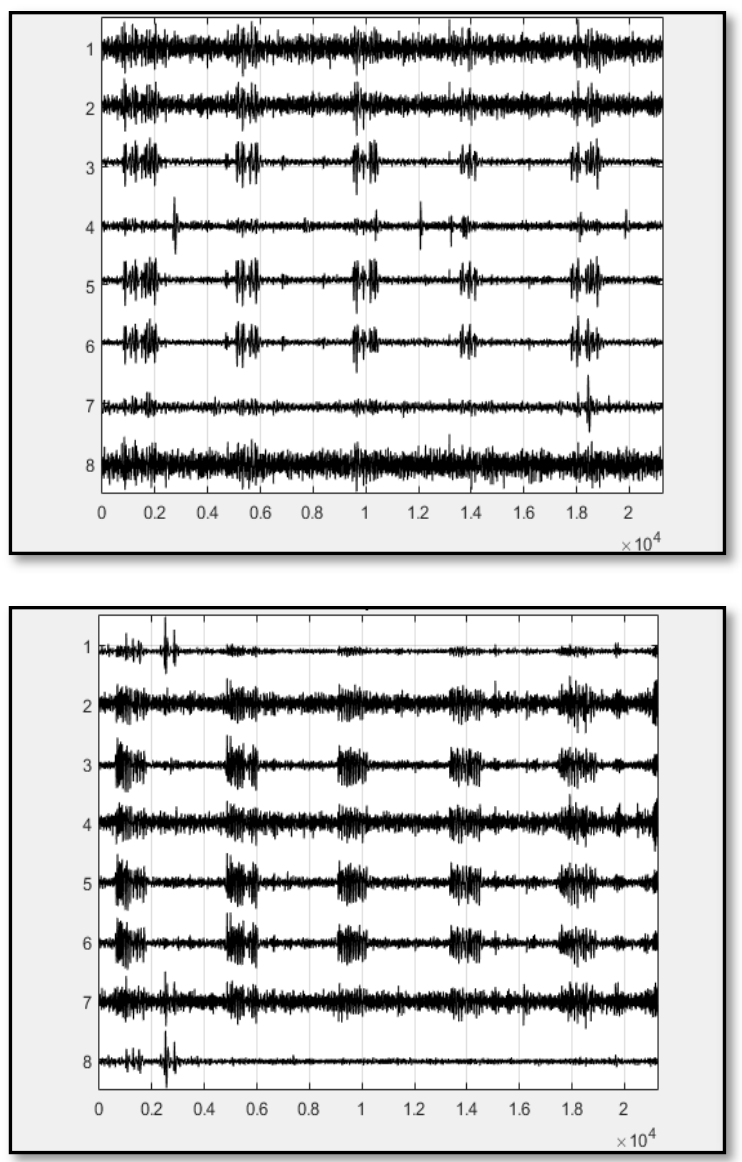

Figura 5. Señales en estado bruto de los datos, de dos participantes diferentes.

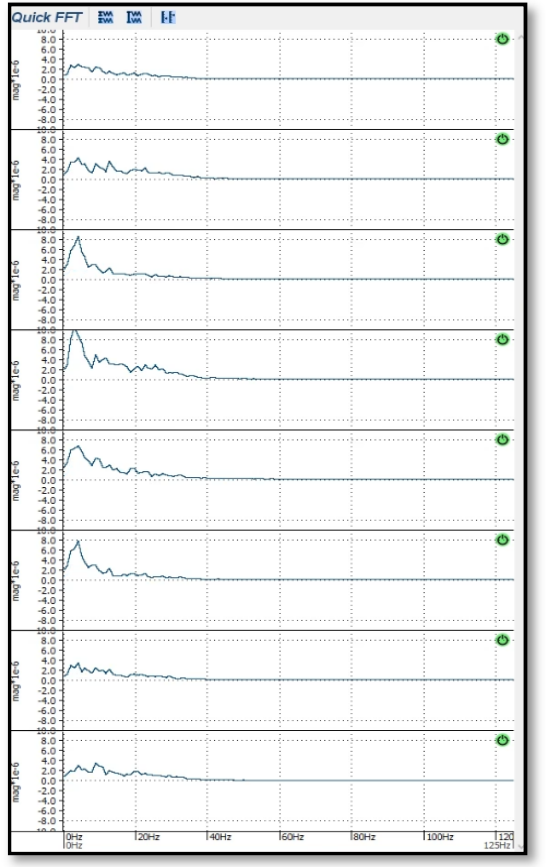

a) Participante en calma con ojos abiertos.

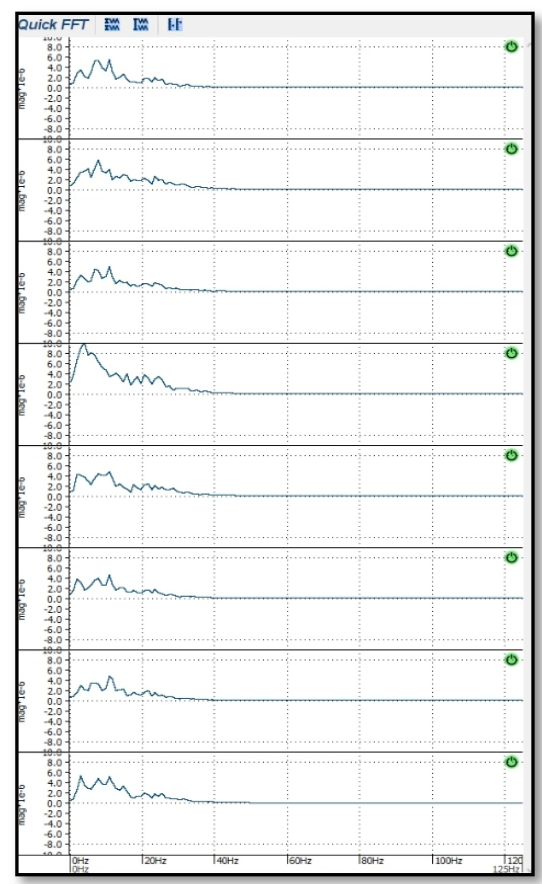

b) Participante en calma con los ojos cerrados.

Figura 6. Señal en calma de un mismo participante. 


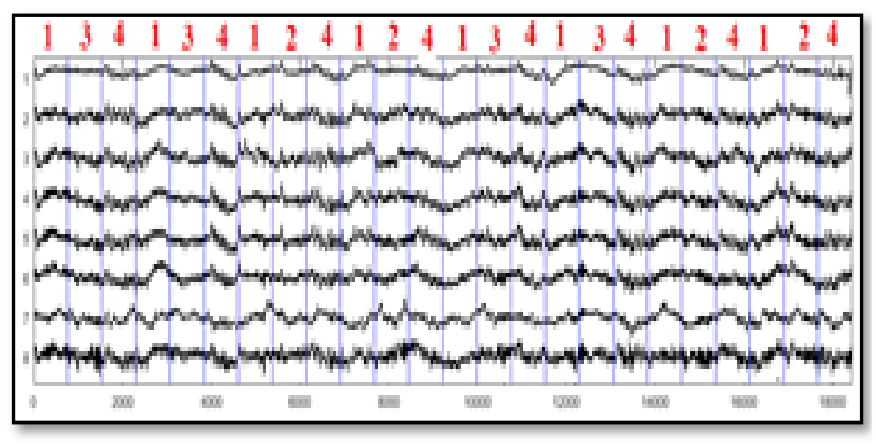

Figura 7. Grupo de señales adquiridas de la imaginación de movimiento de una persona.

Tabla 1. Rangos de las bandas de energía

\begin{tabular}{|c|c|}
\hline Nivel & $\begin{array}{c}\text { Banda de frecuencia } \\
(\mathbf{H z})\end{array}$ \\
\hline 6 & $2-4$ \\
\hline 5 & $4-8$ \\
\hline 4 & $8-16$ \\
\hline 3 & $16-32$ \\
\hline 2 & $32-64$ \\
\hline 1 & $64-128$ \\
\hline
\end{tabular}

Se debe destacar el estado de parpadeo. Este se asignó a manera de control, de tal forma que se pudiese aislar dicha acción solo a estos momentos, y que de esa forma no interviniese con el resto de las señales. Es decir, solo se permitía parpadear en estos instantes. La figura 8 muestra la huella de energía del pensamientoacción del evento de parpadeo. Cabe destacar que esta señal es solo a modo de referencia.

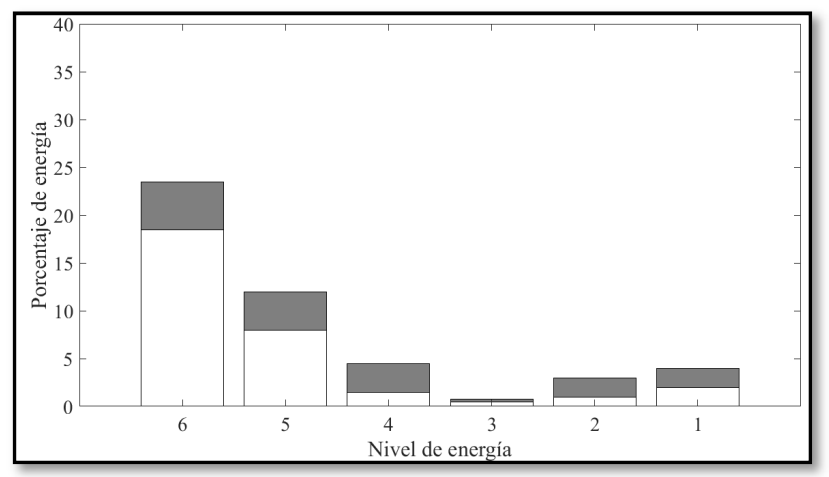

Figura 8. Huella de energía correspondiente al parpadeo. La desviación estándar se muestra en color gris.

El análisis de la señal correspondiente al estado de "descanso" se muestra en la figura 9. La huella de energía se extrajo de las ocho señales EEG del estado de descanso del canal 2 (electrodo $\mathrm{F}_{\mathrm{C} 1}$ ). Este estado originalmente consiste en 768 muestras en el tiempo, pero mediante la aplicación de la técnica, se reduce a una huella de seis muestras, sin pérdida de representatividad.

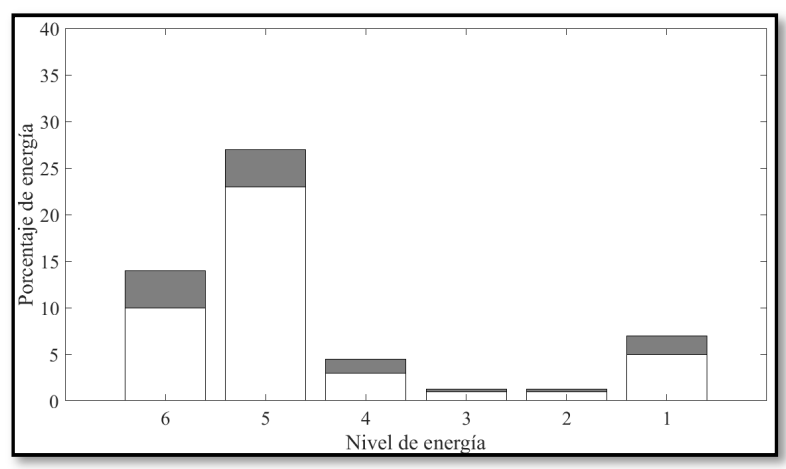

Figura 9. Huella de energía para el pensamiento Descanso. La desviación estándar se muestra en gris para cada nivel.

En la figura 10, se representan las huellas de energía para el pensamiento "derecha" (figura 8a) e "izquierda" (figura 8b).

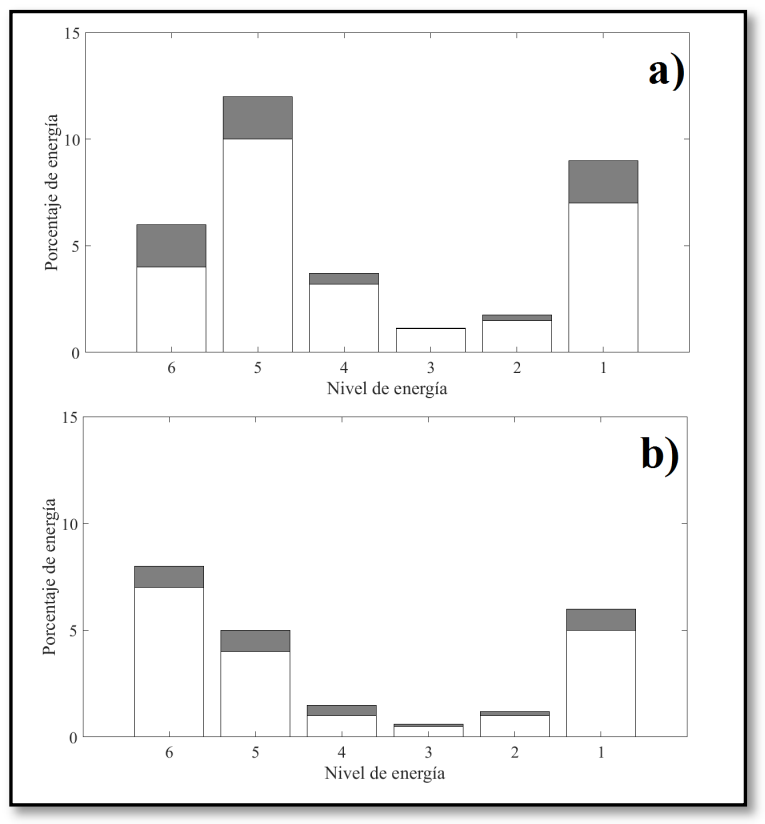

Figura 10. a) Huella de energía para "derecha". b) Huella de energía para "izquierda". Desviaciones estándar en gris.

Para las gráficas de la figura 10 a) y 10 b) se utilizaron solo cuatro señales EEG, tanto para la derecha como para la izquierda. Estas también provenían del canal 2. Es importante agregar, que este mismo análisis 
se realizó para los otros siete canales, presentando diferencias apreciables entre estados de pensamiento. Al observar las huellas de energía para cada pensamiento, se concluye que se puede realizar una clasificación de cada uno de estos estados mediante la técnica propuesta.

\section{Conclusión y discusión}

Los resultados de la señal en su estado bruto demuestran que la señal eléctrica proveniente de los estados del pensamiento del individuo puede ser tratadas con técnicas matemáticas que permiten la caracterización de señales. Para las gráficas de este tipo es necesario realizar más estudios tanto en la utilización de los sensores, con respecto al sistema 10/20, en cuanto a su ubicación; como adquirir datos de otros participantes y comparar los resultados.

Además, los resultados de este estudio indican que la aplicación del método de descomposición de bandas de energía para el análisis de las señales eléctricas obtenidas con un equipo BCI, son posibles; ya que se puede distinguir la banda de energía entre un evento y el otro. Sin embargo, hay que realizar más pruebas para diferenciar claramente entre la imaginación del movimiento de la mano derecha y la imaginación del movimiento de la mano izquierda.

Entre los trabajos futuros referentes al estudio, se pretende hacer una comparación de métodos para el análisis de las señales obtenidas con el equipo BCI, con miras a elegir el mejor método de análisis o una combinación de métodos; así como también controlar dispositivos en el entorno de un hogar, tales como abrir y cerrar puertas.

\section{Agradecimiento}

Agradecimiento a la Secretaría Nacional de Ciencia y Tecnología e Innovación (SENACYT) por financiar el proyecto BCI No 86-2016-4IDDS15-0994, "Desarrollo de un sistema domótico basado en una interfaz cerebromáquina para dar soporte a personas con movilidad reducida"; dentro de la Convocatoria Pública de Fomento a I+D para el desarrollo sostenible (IDDS) 2015.

\section{Referencias}

[1] R. P. N. RAO, Brain-Computer Interfacing: An Introduction, Cambridge: Cambridge University Press, 2013.

[2] J. R. Wolpaw and E. W. Wolpaw, Brain-Computer Interfaces, New York: Oxford.
[3] J.-F. G. A. L. Claude Roberta, "Electroencephalogram processing using neural networks," Clinical Neurophysiology, vol. 113, p. 694-701, 2002.

[4] B. A. a. G. P. Bernhard Graimann, "Brain-Computer Interfaces: A Gentle Introduction," Brain-Computer Interfaces, pp. 1-27, 2010.

[5] F. V. Álvarez, A. F. Rodríguez, S. V. Moya and R. R. Angevin, "Control de una silla de ruedas mediante BCI," in $7^{\circ}$ Simposio CEA de Bioingeniería 2015,Interfaces Cerebro-computador (BCI) y Tecnologías Asistenciales, Málaga, 2015.

[6] J. D. A. Molina and J. A. S. Tipán, Diseño e implementación de un prototipo Brain Computer Interface (BCI), para la manipulacion de una pinza robótica utilizando comunicación bluetooth, Quito, 2014.

[7] J. I. Serrano, M. D. D. Castillo, R. Raya, C. Bayón and E. Rocon, "BCI basado en la facilitación asociativa de la actividad cortical para el inicio de la marcha en Parálisis Cerebral," in $7^{\circ}$ Simposio CEA de Bioingeniería 2015, Interfaces Cerebrocomputador (BCI) y Tecnologías Asistenciales, Málaga, 2015.

[8] R. Hornero, R. Corralejo and D. Álvarez, "Brain-Computer Interface (bCI) aplicado al entrenamiento cognitivo y control domótico para prevenir los efectos del envejecimiento," LYCHNOS, no. 8, pp. 29-34, 2012.

[9] N. U. A. Martínez, "Desarrollo de una interfaz máquina-cerebro con aplicaciones en róbotica médica," Instituto Politécnico Nacional, México, D. F., 2011.

[10] A. Lécuyer, F. Lotte, R. B. Reilly, R. Leeb, M. Hirose and M. Slater, "Brain-computer interfaces, virtual reality, and videogames.," Computer, vol. 41, pp. 66-72, 2008.

[11] R. Navarro, "Instrumentación Biomédica Electroencefalografía. Departamento de Electrónica.," Universidad de Alcalá, Alcalá, España, 1998.

[12] "MatLab," [Online]. Available: https://la.mathworks.com/discovery/wavelet-transforms.html. [Accessed 25 febrero 2018].

[13] E. F. Khalaf, K. Daqrouq and M. Sherif, "Wavelet Packet and Percent of Energy Distribution with Neural Networks Based Gender Identification System," Journal of Applied Sciences, pp. 2940-2946, 2011.

[14] I. Omerhodzic, S. Avdakovic, A. Nuhanovic and K. Dizdarevic, "Energy Distribution of EEG Signals: EEG Signal WaveletNeural Network Classifier".

[15] C. Boya, M. Ruiz-Llata, J. Posada and J. A. Garcia-Souto, "Identification of Multiple Partial Discharge Sources using Acoustic Emission Technique and Blind Source Separation," IEEE Transactions on Dielectrics and Electrical Insulation, vol. 22, pp. 1663-1673.

[16] MathWorks, "Análisis Wavelet de 1-D MultiSignal," [Online]. Available: https://www.mathworks.com/help/wavelet/examples/multisignal1-d-wavelet-analysis.html. [Accessed 25 Julio 2018].

[17] J. M. Morales, S. Romero, L. L. D. Stasi and C. Diaz-Piedra, "Plataforma para la monitorización de bioseñales en conductores," in $7^{\circ}$ Simposio CEA de Bioingeniería 2015, Interfaces Cerebro-computador (BCI) y Tecnologías Asistenciales, Málaga, 2015. 
[18] A. Nijholt, R. J. Jacob, M. Andujar, B. F. Yuksel and G. Leslie, "Brain-Computer Interface for Artistic Expression," 2018.

[19] I. Kosunen, A. Ruonala, M. Salminen, S. Järvelä, N. Ravaja and G. Jacucci, "Neuroadaptive Meditation in the Real World," BCIforReal '17 Proceedings of the 2017 ACM Workshop on An Application-oriented Approach to BCI out of the laboratory, pp. 29-33, Marzo 2017.
[20] A. Al-Cahalabi, M. Turner and S. Delamont, The Brain, New York: Oneworld Publications, 2006.

[21] S. J., "Técnicas de toma de datos y análisis de Electroencefalografía.," Universidad de Sevilla, Sevilla, España, 2014. 